25. Li L, Zhang W, Hu Y, et al. Effect of convalescent plasma therapy on time to clinical improvement in patients with severe and life-threatening COVID-19: a randomized clinical trial. JAMA. 2020;324(5):460470 .

26. Liu Z. Errors in trial of effect of convalescent plasma therapy on time to clinical improvement in patients with severe and life-threatening COVID-19. JAMA. 2020;324(5):518-519.

27. Casadevall A, Joyner MJ, Pirofski LA. A randomized trial of convalescent plasma for COVID-19-potentially hopeful signals. JAMA. 2020;324(5):455-457.

28. Zhang B, Liu S, Tan T, et al. Treatment with convalescent plasma for critically Ill patients with severe acute respiratory syndrome coronavirus 2 infection. Chest. 2020;158(1):e9-e13.

29. Duan K, Liu B, Li C, et al. Effectiveness of convalescent plasma therapy in severe COVID-19 patients. Proc Natl Acad Sci U S A. 2020;117(17):9490-9496.

30. Ahn JY, Sohn Y, Lee SH, et al. Use of convalescent plasma therapy in two COVID-19 patients with acute respiratory distress syndrome in Korea. J Korean Med Sci. 2020;35(14):e149.

31. Ye M, Fu D, Ren Y, et al. Treatment with convalescent plasma for COVID-19 patients in Wuhan, China. J Med Virol. 2020 Apr 15. [Epub ahead of print]

32. Shen C, Wang Z, Zhao F, et al. Treatment of 5 critically Ill patients with COVID-19 with convalescent plasma. JAMA. 2020;323(16):1582-1589.
33. Zeng OL, Yu ZJ, Gou JJ, et al. Effect of convalescent plasma therapy on viral shedding and survival in patients with coronavirus disease 2019. J Infect Dis. 2020;222(1):38-43.

34. Murphy M, Estcourt L, Grant-Casey J, Dzik S. International survey of trials of convalescent plasma to treat COVID-19 infection. Transfus Med Rev. 2020;34(3):151-157.

35. Joyner MJ, Bruno KA, Klassen SA, et al. Safety update: COVID-19 convalescent plasma in 20,000 hospitalized patients. Mayo Clin Proc. 2020;95(9):1888-1897.

36. Birra D, Benucci M, Landolfi L, et al. COVID 19: a clue from innate immunity. Immunol Res. 2020;68(3):161-168.

37. Gralinski LE, Sheahan TP, Morrison TE, et al. Complement activation contributes to severe acute respiratory syndrome coronavirus pathogenesis. mBio. 2018;9(5): e01753-18.

38. Al-Samkari H, Karp Leaf RS, Dzik WH, et al. COVID-19 and coagulation: bleeding and thrombotic manifestations of SARS-CoV-2 infection. Blood. 2020;136(4):489-500.

39. Dzik S. Complement and coagulation: cross talk through time Transfus Med Rev. 2019;33(4):199-206.

40. Dzik S. COVID-19 convalescent plasma: now is the time for better science. Transfus Med Rev. 2020;34(3):141-144.

41. Li L, Tong X, Chen $\mathrm{H}$, et al. Characteristics and serological patterns of COVID-19 convalescent plasma donors: optimal donors and timing of donation. Transfusion. $2020 \mathrm{Jul} 6$. [Epub ahead of print]

\title{
Expanding dasatinib beyond KIT in acute myeloid leukemia
}

\author{
John S. Welch \\ Washington University School of Medicine, St. Louis, MO, USA \\ E-mail: JOHN S.WELCH - jwelch@wustl.edu
}

doi:10.3324/haematol.2020.262147

T The goal of personalized medicine is to match patient-specific factors with relevant therapeutic options. The therapeutic conundrum in acute myeloid leukemia (AML) remains the heterogeneity of the disease and the paucity of treatment options for which there are highly predictive biomarkers. AML is broadly heterogeneous across all measured axes, including morphological presentation, cytogenetics, point mutations, expression signatures, epigenetic signatures, and chromatin signatures. ${ }^{1,2}$ Furthermore, within patients, subclonal architecture suggests ongoing acquisition of new variants and expression signatures, ${ }^{3}$ providing for intrapatient leukemic heterogeneity as well as interpatient heterogeneity. Just as heterogeneity can be seen across a range of measurements, response diversity has been mapped to clinical and molecular diversity, providing prognostic opportunities, but not yet personalized opportunities. ${ }^{2}$

AML results in unrestrained growth of the leukemia cells in vivo, but this has not translated into easy in vitro growth sufficient for effective cell manipulation in laboratory settings. Primary human AML cells grow poorly in liquid tissue culture media or methylcellulose. Only a fraction of samples will effectively engraft into immunodeficient mice, and among these, it is often only a subclone that engrafts. ${ }^{4}$ Recent progress has made short-term ex vivo culture possible, and improvements in the immunodeficient hosts have improved xenograft potential. ${ }^{5}$

Ex vivo analysis of chemotherapy has been championed by several groups, including the large-scale BEAT AML project. ${ }^{6.9}$ Studies have increasingly suggested ex vivo corre- lations with clinical response and the feasibility of scaling up to achieve sufficient throughput to identify useful functional biomarkers of sensitivity and resistance to chemotherapy.

In this issue, Tavor et al. present a focused analysis that leverages careful sample selection with ex vivo drug sensitivity. ${ }^{10}$ They applied a 384-well approach to interrogates cell viability in liquid culture after 48 hours assessed across 46 drugs, each at 12 concentrations, which provided a broad area under the curve (AUC) measurement. In this assay, the authors used cytokine combinations (colonystimulating factor, interleukin 3, interleukin 6, thrombopoietin) in liquid culture and avoided stromal cell co-culture to facilitate viability read-out using a streamlined ATP-dependent assay (Cell Titer Glo). This approach provides an efficient read-out of early chemotoxicity, but does not provide an effective measure of differentiation or the cell toxicity that occurs after several days of exposure or cell divisions. In evaluating outcomes using this design, it is worth noting that the strong cytokine stimuli in the tissue culture may bias cell survival and chemosensitivity to cells that are capable of utilizing those signaling pathways or dependent on their stimuli for survival, and the small cell numbers evaluated in 384-well formats focus outcomes on phenotypes in the bulk cell population.

Tavor et al. found that relapse samples were less chemosensitive than the paired diagnostic sample, across diverse classes of chemotherapy. Indeed, there were statistical differences between the sensitivity of diagnostic and relapse pairs to some common salvage agents, including 
etoposide and fludarabine, while cytarabine and venetoclax were associated with strong trends. This phenomenon of reduced sensitivity at relapse, compared with diagnosis, has played out across diverse chemotherapy regimens and newer venetoclax-based approaches in vivo. ${ }^{11,12}$ This provides a possible clinical validation of the platform and insight into relapsed AML as a broadly chemoresistant disease and not simply a cytarabine-resistant problem.

Although the sample size was relatively small (29 patients), sensitivity and resistance could be clustered by functional groups, with tyrosine kinase inhibitors emerging with shared phenotypes. Drug targets included MEK, PI3K, PIM, JAK, mTORC1/2, FLT3, and BET inhibitors.

To understand the mechanism of activity and to expand potential biomarker targets, Tavor et al. integrated mutation and transcriptome data with the functional chemosensitivity results. This identified increased frequency of FLT3-ITD variants in the chemosensitive cases. In particular, dasatinib emerged as a compound for which there were wide differences in chemosensitivity between these groups of patients. The authors further leveraged available results from the BEAT-AML study with their transcriptional data to identify overlapping signatures associated with dasatinib sensitivity, including dasatinib targets $(C S F 1 R$, $F G R, H C K$, and $L Y N$ ). Using further model building and validation between the two cohorts, a model with an AUC 0.78 could be generated, providing an intriguing new biomarker for dasatinib. Finally, response to dasatinib could be observed in xenograft models of FLT3-ITD AML.

Dasatinib has been studied in combination with multichemotherapy regimens in both acute lymphoblastic leukemia and AML, ${ }^{13-15}$ and thus is a natural compound to consider for quick integration into biomarker-driven AML therapy. In AML, the interest in dasatinib has focused on its use for the core-binding factor leukemias (CBF; $t(8 ; 21)$ and inv(16)), which are frequently associated with mutations in KIT, and where the presence of the secondary KIT mutation is associated with inferior outcomes. ${ }^{16}$ Dasatinib has been explored in single-arm studies by the Cancer and Leukemia Group B (CALGB) and the Acute Myeloid Leukemia Study Group (AMLSG) to determine whether KIT inhibition could mitigate the negative effects of these variants. In both studies, responses and survival were favorable compared with those of historical controls. In the CALGB study, the adverse impact of KIT mutations appeared to have been effaced by dasatinib treatment. ${ }^{13}$ Analysis in the AMLSG study included paired evaluation of nine patients with KIT mutations. Five of these patients lost the variant at relapse, suggesting a potential selective disadvantage in the presence of dasatinib..$^{15}$

In contrast to the molecular focus of dasatinib as an antiKIT agent in the CBF leukemia studies, Tavor et al. identified broader kinase mutations and kinase activity that correlate with dasatinib sensitivity, suggesting that additional biomarkers may be relevant for predicting sensitivity to dasatinib in non-CBF AML. ${ }^{10}$

In AML, biomarker-driven personalized medicine is challenged by the therapeutic timeline. Treatment needs to begin quickly once the diagnosis has been made. However, the delays in therapy initiation appear to affect predominantly younger patients, ${ }^{17}$ and the RADIFY study suggested that a delay of 8 days in adding a tyrosine kinase inhibitor to induction chemotherapy did not compromise efficacy in the younger age group..$^{18}$ This provides a reasonable window for either ex vivo drug screening to be completed or mutation and transcriptomic analysis to be finalized while chemotherapy options are fine-tuned.

AML is diversely heterogeneous between patients, but outcomes will be patient-specific. The application of ex vivo drug screening and the integration of results with

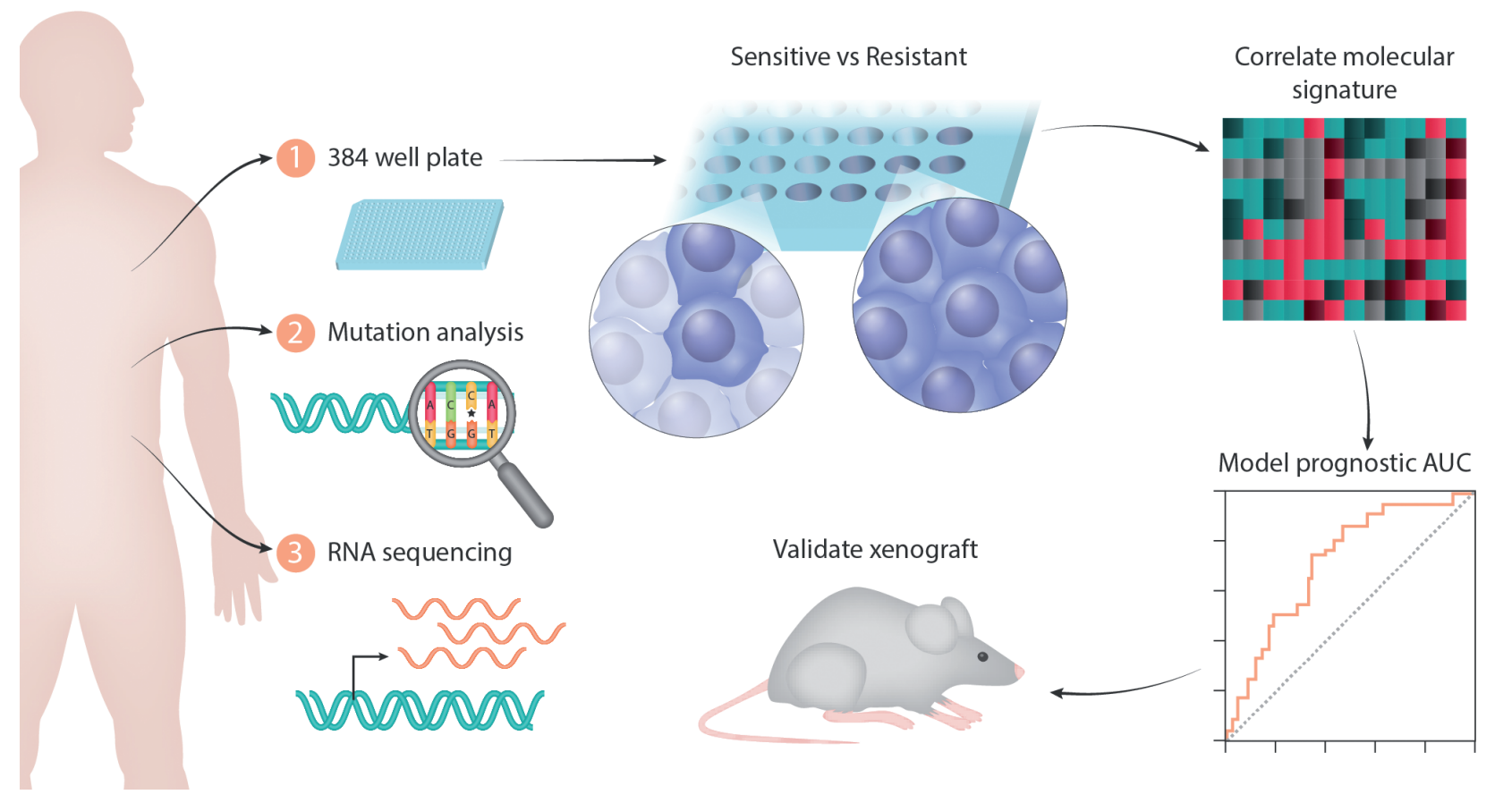

Figure 1. Integration of ex vivo drug sensitivity data and molecular signatures described in Tavor et al. ${ }^{10}$ AUC: area under the curve. 
expression and mutation signatures may provide the means of ultimately matching patients with treatment and matching treatment with response mechanisms. Given that relapsed disease appears to be chemoresistant across multiple classes of therapy, integration of personalized treatment is likely to be most effective when applied as early as possible.

\section{References}

1. Cancer Genome Atlas Research Network. Genomic and epigenomic landscapes of adult de novo acute myeloid leukemia. N Engl J Med. 2013;368(22):2059-2074.

2. Papaemmanuil E, Gerstung M, Bullinger L, et al. Genomic classification and prognosis in acute myeloid leukemia. N Engl J Med. 2016;374(23):2209-2221.

3. Welch JS, Ley TJ, Link DC, et al. The origin and evolution of mutations in acute myeloid leukemia. Cell. 2012;150(2):264-278.

4. Klco JM, Spencer DH, Miller CA, et al. Functional heterogeneity of genetically defined subclones in acute myeloid leukemia. Cancer Cell. 2014;25(3):379-392.

5. Krevvata M, Shan X, Zhou C, et al. Cytokines increase engraftment of human acute myeloid leukemia cells in immunocompromised mice but not engraftment of human myelodysplastic syndrome cells. Haematologica. 2018;103(6):959-971.

6. Tyner JW, Tognon CE, Bottomly D, et al. Functional genomic landscape of acute myeloid leukaemia. Nature. 2018;562(7728):526-531.

7. Klco JM, Spencer DH, Lamprecht TL, et al. Genomic impact of transient low-dose decitabine treatment on primary AML cells. Blood. 2013;121(9):1633-1643.

8. Kurtz SE, Eide CA, Kaempf A, et al. Molecularly targeted drug combi- nations demonstrate selective effectiveness for myeloid- and lymphoid-derived hematologic malignancies. Proc Natl Acad Sci U S A. 2017;114(36):E7554-E7563.

9. Malani D, Murumagi A, Yadav B, et al. Enhanced sensitivity to glucocorticoids in cytarabine-resistant AML. Leukemia. 2017;31(5):11871195.

10. Tavor S, Shalit T, Ilani NC, et al. Dasatinib response in acute myeloid leukemia is correlated with FLT3/ITD, PTPN11 mutations and a unique gene expression signature. Haematologica. 2020;105(12):27952804.

11. Estey EH. Treatment of relapsed and refractory acute myelogenous leukemia. Leukemia. 2000;14(3):476-479.

12. DiNardo CD, Rausch CR, Bent on C, et al. Clinical experience with the BCL2-inhibitor venetoclax in combination therapy for relapsed and refractory acute myeloid leukemia and related myeloid malignancies. Am J Hematol. 2018;93(3):401-407.

13. Marcucci G, Geyer S, Laumann K, et al. Combination of dasatinib with chemotherapy in previously untreated core binding factor acute myeloid leukemia: CALGB 10801. Blood Adv. 2020;4(4):696-705.

14. Ravandi F, O'Brien S, Thomas D, et al. First report of phase 2 study of dasatinib with hyper-CVAD for the frontline treatment of patients with Philadelphia chromosome-positive $(\mathrm{Ph}+)$ acute lymphoblastic leukemia. Blood. 2010;116(12):2070-2077

15. Paschka P, Schlenk RF, Weber D, et al. Adding dasatinib to intensive treatment in core-binding factor acute myeloid leukemia-results of the AMLSG 11-08 trial. Leukemia. 2018;32(7):1621-1630.

16. Ayatollahi H, Shajiei A, Sadeghian MH, et al. Prognostic importance of C-KIT mutations in core binding factor acute myeloid leukemia: a systematic review. Hematol Oncol Stem Cell Ther. 2017;10(1):1-7.

17. Sekeres MA, Elson P, Kalaycio ME, et al. Time from diagnosis to treatment initiation predicts survival in younger, but not older, acute myeloid leukemia patients. Blood. 2009;113(1):28-36.

18. Stone RM, Mandrekar SJ, Sanford BL, et al. Midostaurin plus Chemotherapy for acute myeloid leukemia with a FLT3 mutation. N Engl J Med. 2017;377(5):454-464.

\section{Convalescent plasma for administration of passive antibodies against viral agents}

\section{Giovanni Di Minno, ${ }^{1}$ Pier Mannuccio Mannucci, ${ }^{2}$ James W. Ironside, ${ }^{3}$ Carlo Federico Perno, ${ }^{4}$ Lutz Gürtler ${ }^{5}$ and Louis Aledort ${ }^{6}$}

${ }^{1}$ Dipartimento di Medicina Clinica e Chirurgia, Centro Hub per le Emocoagulopatie, Napoli, Italy; ${ }^{2}$ Fondazione IRCCS Ca' Granda Ospedale Maggiore Policlinico, Angelo Bianchi Bonomi Hemophilia and Thrombosis Center, Milan, Italy; ${ }^{3}$ Centre for Clinical Brain Sciences, University of Edinburgh, Edinburgh, UK; ${ }^{4}$ Departmental Medicine Laboratory, ASST Niguarda Hospital, Milan, Italy; ${ }^{5}$ Max von Pettenkofer Institute, Ludwig Maximilians University of Munich, Munich, Germany and 'Mary Weinfeld Professor of Clinical Research in Hemophilia at the Icahn School of Medicine at Mount Sinai, New York City, NY, USA

E-mail: GIOVANNI DI MINNO - diminno@unina.it

doi:10.3324/haematol.2020.267427

A dministration of passive antibodies through transfusion of plasma from donors recovering from a viral infection has long been employed to treat individuals infected with the same pathogen. ${ }^{1}$ However, in studies with convalescent plasma (CP), differences and inherent limitations (e.g., sensitivity/specificity of tests to quantify neutralizing antibodies; sample size; scheduling of treatment [early/late CP administration vs. degree of disease severity], the presence of confounders [concomitant treatments]), and restricted generalizability of data argued for large-scale, randomized, controlled trials. ${ }^{1,2}$ The results of a multicenter proof-of-concept, observational Italian study in 46 patients with moderate or severe acute respiratory distress syndrome due to infection with the novel coronavirus, SAR-CoV-2, who needed mechanical ventilation and/or continuous positive airway pressure are reported in this issue of the Journal. ${ }^{3}$ The interval between symptom onset and study inclusion was highly variable (2-29 days). The 7 day mortality rate was $6 \%$ in patients given CP compared with an expected $15 \%$ according to Italian statistics and $30 \%$ in a small concurrent cohort not treated with CP. Weaning from continuous positive airway pressure was achieved in 26 of 30 patients, and three of the seven intubated patients were extubated. Whether those who received $\mathrm{CP}$ earlier improved more or faster than patients who received plasma later in the course of the disease is not clarified, nor are the reasons for administering one, two or three CP bags provided. In this larger than previous uncontrolled reports, five serious adverse events (including 1 transfusion-related acute lung injury [TRALI]) occurred in four patients. Although TRALI may be triggered by transfused antibodies, ${ }^{4}$ CP was safe in this study as it was in 